\title{
Fuzzy Based Intelligent System to Predict Most Suitable Crop
}

\author{
R. Joshi ${ }^{1}$, H. Fadewar ${ }^{2}$, P. Bhalchandra ${ }^{2}$ \\ ${ }^{1}$ Department of Computer Sceicne, Yogeshwari College, Ambajogai, Dist. Beed \\ ${ }^{2}$ School of Computational Sciecnes, S.R.T.M.University, Nanded, MS, 431606, India \\ \{yma.joshi@gmail.com, fadewar_hsf@yahoo.com,srtmun.parag@gmail.com\}
}

\begin{abstract}
This paper describes design, development and deployment of a fuzzy based agricultural decision support system which help farmers to make wise decisions regarding selection of a crop. Various parameters for decision making are understood and Fuzzy interface systems are developed. This study finds application in India as the selection of crop is based on folklore knowledge and farmers find this process extremely hard. Choosing most suitable crop is the major problem farmer has to face.
\end{abstract}

Keywords: DSS, Data Mining, Analysis of Patterns, Fuzzy Logic

\section{Introduction}

Indian economy is largely dependent on agriculture as the agriculture alone contributes $75 \%$ of Indian jobs. The most difficult problem Indian farmer face is selection of crop keeping in mind environmental conditions and economic gains. The tradition process for selection of crops is totally based on folklore knowledge and historical facts. In entire farming process, taking a good decision at proper time directly affects the total production of crop [5]. We have tried to make this process scientific by coming with a decision support system. During design and development process, we have observed that a good decision comes from analysis of number of parameters like rainfall, type of soil, temperature, humidity, chemical composition of soil etc. Almost all parameters are uncertain. That's why we have incorporated fuzzy aspects for handling uncertainty. This fuzzy DSS can give most suitable solutions [4]. The fuzzy aspects are incorporated using fuzzy logic tool box available in MATLAB. Our system is a rule based system fuzzy DSS.

\section{Fuzzy Inference System}

The Fuzzy Inference Systems (FIS) are popular computing frameworks based on the concepts of fuzzy set theory, which have been applied with success in many fields [1,2,3]. Their success is mainly due to their closeness to human perception and reasoning, as well as their intuitive handling and simplicity, which are important factors for acceptance and usability of the systems.

In particular, three main modules are of particular interest: a fuzzifier, a rule base and a defuzzifier. While the fuzzifier and the defuzzifier has the role of converting external information in fuzzy quantities and vice versa, the core of a FIS is its knowledge base, which is expressed in terms of fuzzy rules and allows for approximate reasoning.

Our literature review suggests that the Mamdani FIS type [2,3] was proposed as the first attempt to solve control problems by a set of linguistic rules obtained from experienced human operators. The main feature of such type of FIS is that both the antecedents and the consequents of the rules are expressed as linguistic constraints. As a consequence, a Mamdani FIS can provide a highly intuitive knowledge base that is easy to understand and maintain, though its rule formalization requires a time consuming Defuzzification procedure. For such reasons, Mamdani type FISs can be used as valid supports in all such fields - like Medicine, Economics, etc. - where transparency more than cutting-edge efficiency is required.

There are also Rule based System. A rule-based system consists of if-then rules, a bunch of facts, and an interpreter controlling the application of the rules, given the facts. These if-then rule statements are used to formulate the conditional statements that comprise the complete knowledge base [7].

B. Iyer, S. Nalbalwar and R. Pawade (Eds.)

ICCASP/ICMMD-2016. Advances in Intelligent Systems Research.

Vol. 137, Pp. 380-384.

(C) 2017- The authors. Published by Atlantis Press

This is an open access article under the CC BY-NC license (http://creativecommons.org/licens)es/by-nc/4.0/). 
A single if-then rule assumes the form 'if $\mathrm{x}$ is $\mathrm{A}$ then $\mathrm{y}$ is $\mathrm{B}$ ' and the if-part of the rule ' $\mathrm{x}$ is $\mathrm{A}$ ' is called the antecedent or premise, while the then-part of the rule ' $\mathrm{y}$ is $\mathrm{B}$ ' is called the consequent or conclusion. There are two broad kinds of inference engines used in rule-based systems: forward chaining and backward chaining systems. In a forward chaining system, the initial facts are processed first, and keep using the rules to draw new conclusions given those facts [8]. In a backward chaining system, the hypothesis (or solution/goal) we are trying to reach is processed first, and keep looking for rules that would allow concluding that hypothesis. We have designed our fuzzy DSS using Mamdani FIS and Rule based systems.

\section{System Development}

The proposed system is designed and developed by using GUI and Fuzzy logic tool box of MATLAB. For proper designing of system, we have extensively studied earlier similar works. Basic important parameters are identified from earlier work and then new system is designed with additional parameters which are not considered by earlier researchers. Our system has 15 input parameters. We use 'gauss $2 \mathrm{mf}^{\prime}$ membership function for fuzzification of input and 'trimf' membership function is used for fuzzification of output[6]. There are 22 rules in the system. There are five stages of the fuzzy inference process.

1. Fuzzification of the input variables

2. Application of the fuzzy operator (AND or OR) in the antecedent

3. Implication from the antecedent to the consequent,

4. Aggregation of the consequents across the rules

5. Defuzzification

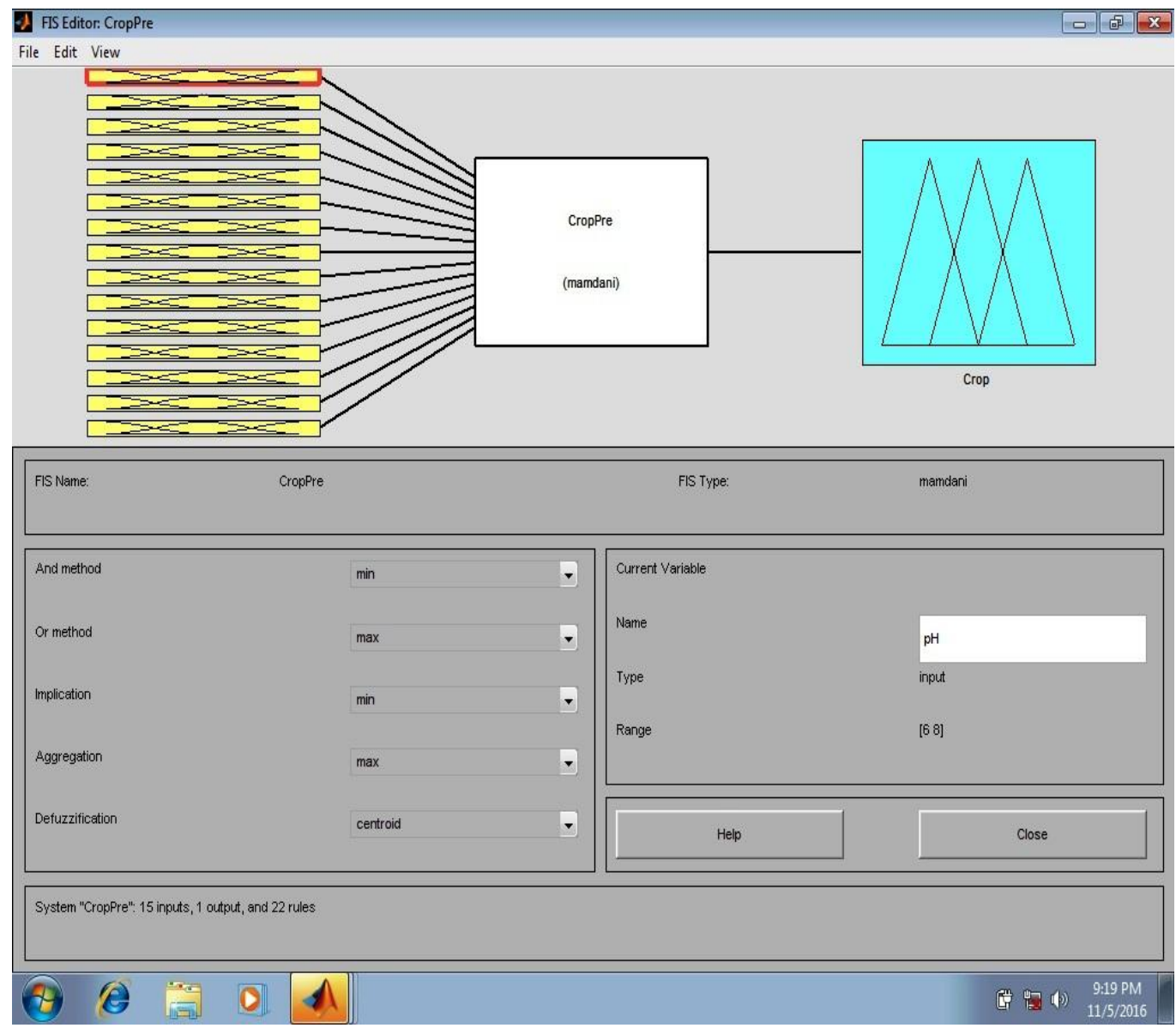

Fig.1. FIS Editor (Mamdani Model)

The FIS structure is the MATLAB object that contains all the fuzzy inference system information. The FIS structure is prepared for Mamdani model. This model has 15 inputs. One output and 22 rule base. For 
fuzzification of soil parameters " gaussmf " function is used. For fuzzification of output " trimf " function is used.22 Rule base are prepared.

This model, by considering the rule base, gives one output crop which will provide the maximum yield. To get the output in a crisp value Defuzzification is done using centroid method.

The following figures show demonstration of developed software system using fuzzy logic tool box of MATLAB.

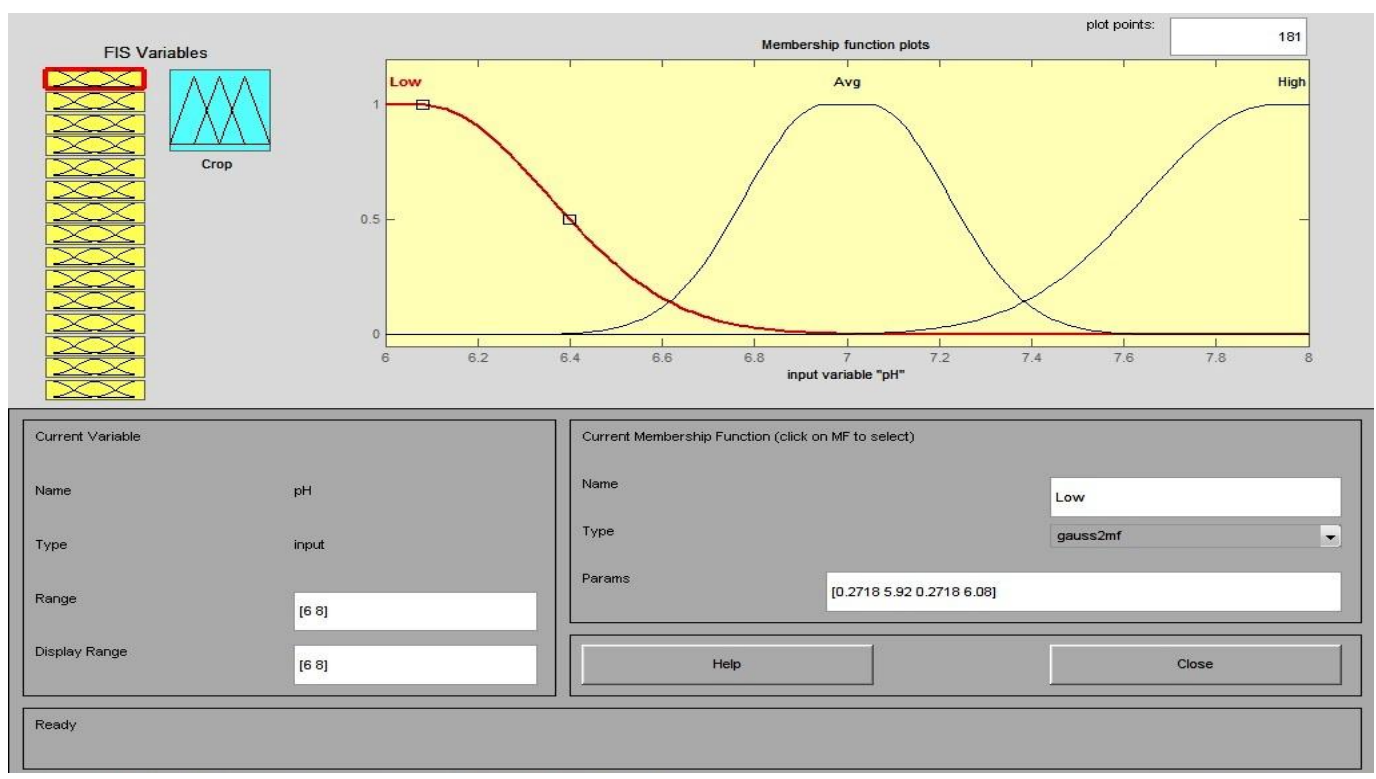

Fig.2. Membership function editor

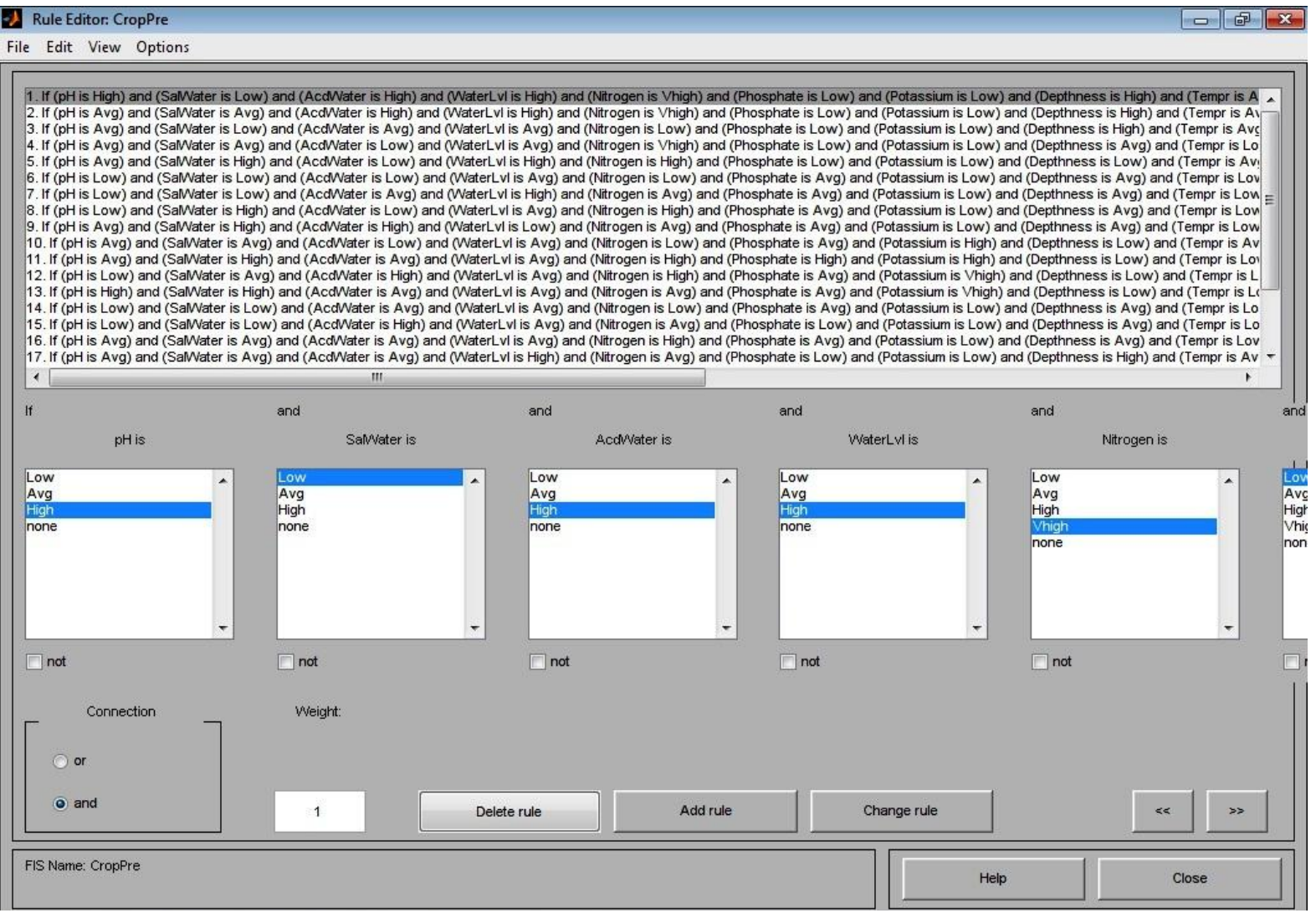

Fig.3. Rule editor for crop

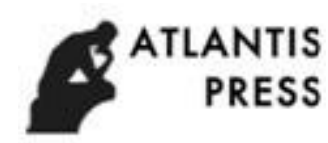




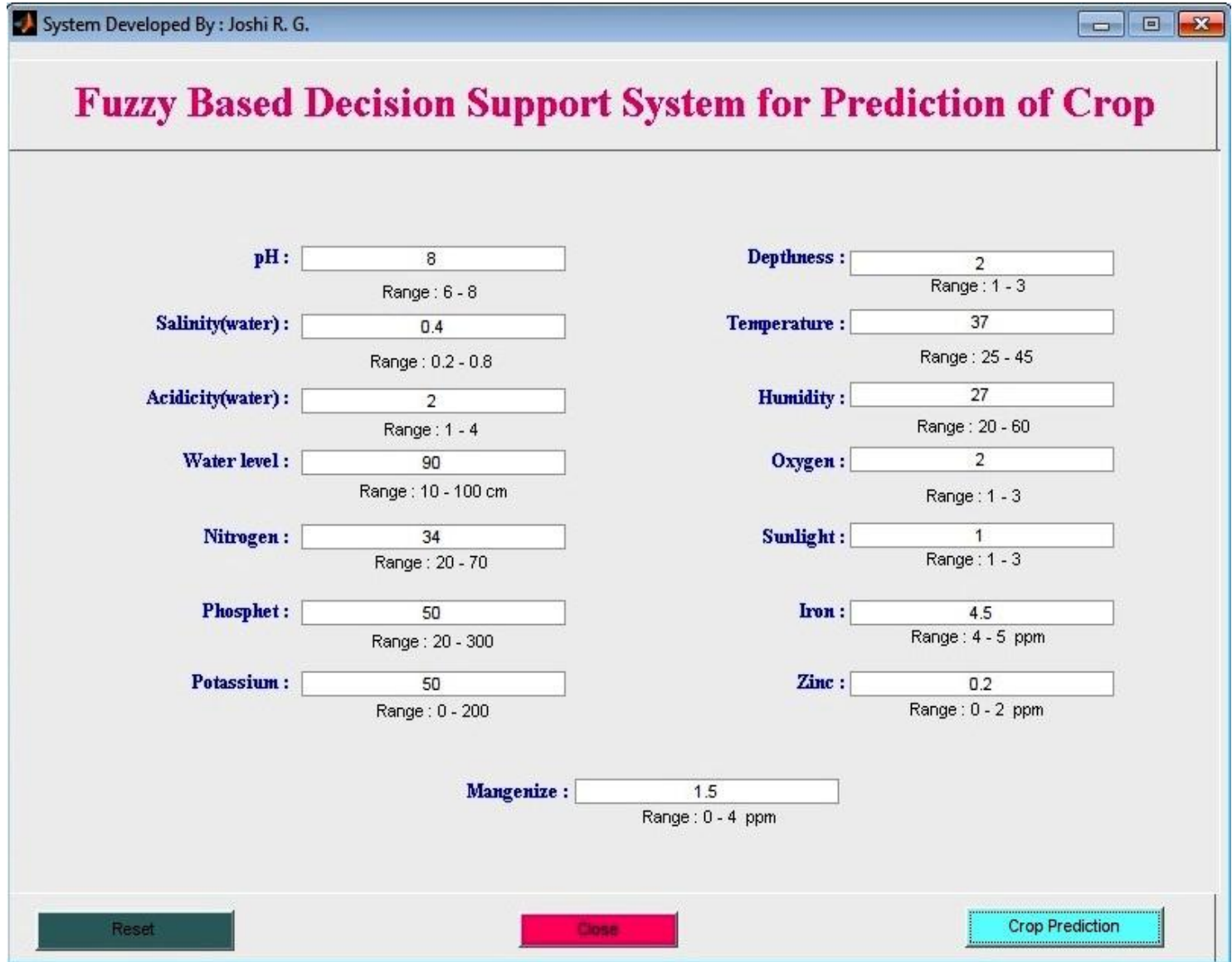

Fig.4. Input given to system.

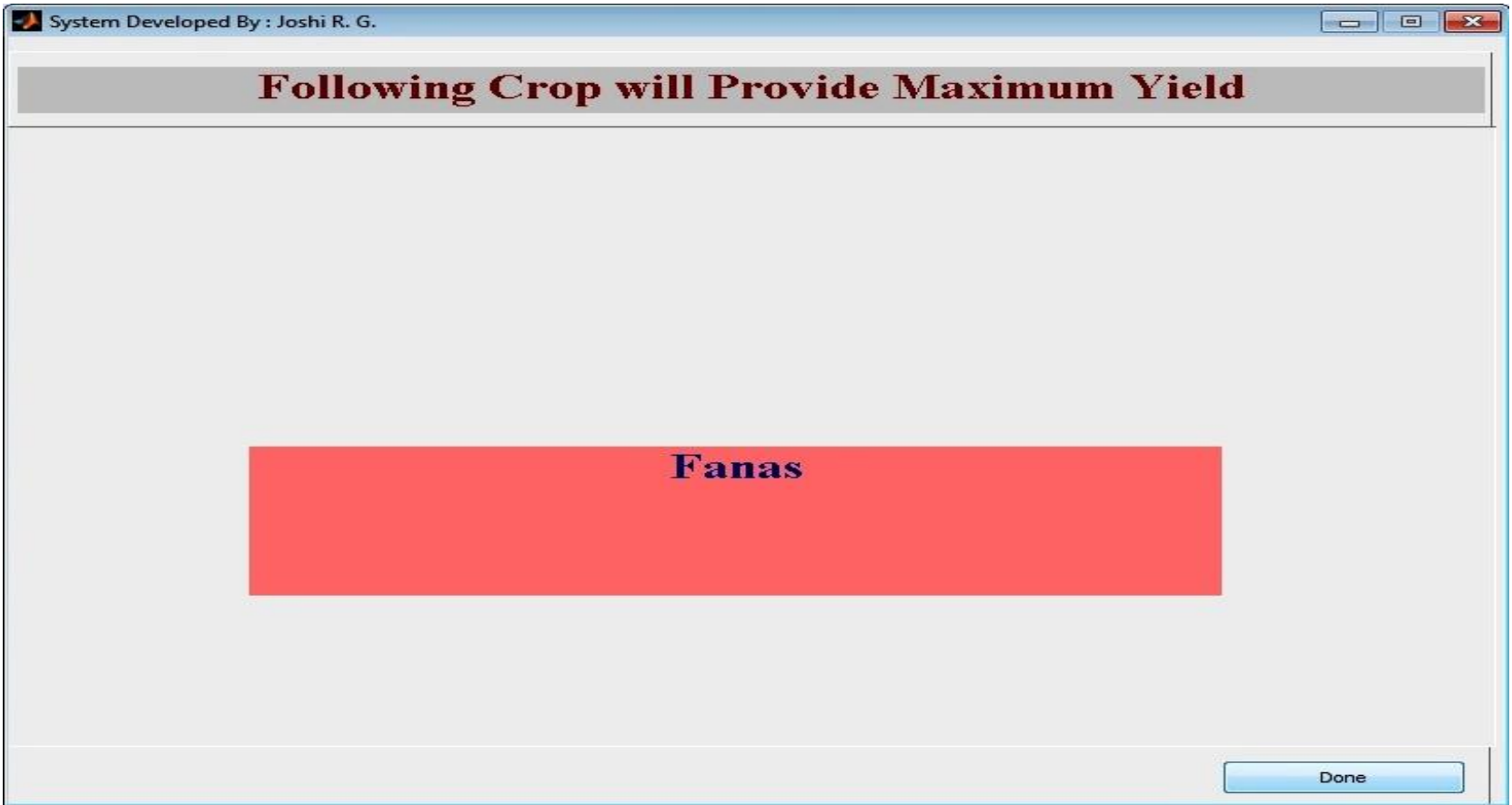

Fig.5. Output of the system 


\section{System Demo for a Crop}

User must provide the necessary parameters for the selection of suitable crop. For user convenience, reference value for each parameter is shown on user screen. For example range of temperature lies in between 25-45 degree centigrade. Similarly the range of earth elements/parameter such as phosphate, potassium, nitrogen, humidity etc. are to be inputted to system. The rules described in FIS file system will automatically provide most suitable crop for the given parameter.

\section{Conclusion}

This paper summarizes design, development and deployment of a fuzzy based agriculture system which provides a novel application of fuzzy logic in the field of agriculture. This system has largely reduces the burden of farmer by proposing a scientific method for selecting best suitable crop among the list of crops. Our work has considered 15 parameters, which are maximum among contemporary works and 22 different fuzzy rules. This extensive framework gives good results. The system is deployed at many places and results are found to be accurate. This paper draws its conclusion from the experimental observations.

\section{References}

[1] Ying Bai, Hanqi Zhuang and Zvi. S Roth, : Fuzzy Logic Control to Suppress Noisesand Coupling Effects in a Laser Tracking System, IEEE Trans on Control Systems Technology, Vol.13, No.1, pp.113-121 (January 2005)

[2] Philomine Roseline, et al, :A Study of Applications of Fuzzy Logic in Various Domains of Agricultural Sciences ,International Journal of Computer Applications (0975 - 8887) International Conference on Current Trends in Advanced Computing (ICCTAC-2015)

[3] Debrup Chakraborty and Nikhil R. Pal ,:A Neuro-Fuzzy Scheme for Simultaneous Feature Selection and Fuzzy Rule-Based Classification, IEEE Transation on Neural Networks, Vol 15 (2004)

[4] Shaofeng et al, : Integration of decision support systems to improve decision support performance, Springer-verlag,London (2009)

[5] M.N.Reddy, N.H.Rao,:MIS based Decision Support System in Agriculture, Indian agriculture development conference (2006)

[6] Web resource at www.mathworks.com

[7] V.O. Oladokun, D.I. Oyewale, : A Fuzzy Inference Based DSS for Solving the University-Course Admission Choice Problem, International Journal of computer Application, Vol. 112 (2015)

[8] Kunjal Mankad, et al, :Evolving Rule Using Genetic Fuzzy Approach-An Educati0nal Case Study, International Journal on Soft Computing Vol 2 (2011)

[9] M. Patil, B. Iyer and R. Arya, "Performance Evaluation of PCA and ICA Algorithm for Facial Expression Recognition Application", Proceedings of Fifth International Conference on Soft Computing for Problem Solving, vol.436, 965-976 (2016). 